\title{
Beilinson and Drinfeld Awarded 2018 Wolf Prize in Mathematics
}

ALEXANDER BEILINSON and VLADIMIR DRINFELD of the University of Chicago have been awarded the Wolf Foundation Prize for Mathematics for 2018 by the Wolf Foundation.

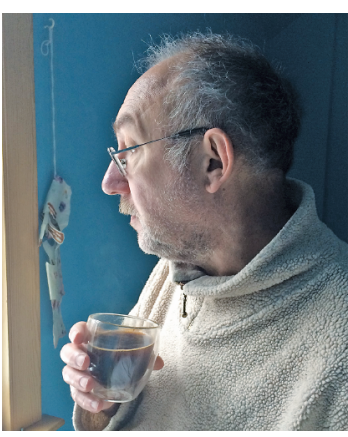

Alexander Beilinson

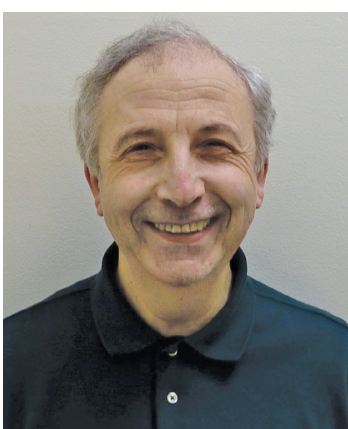

Vladimir Drinfeld
The prize citation reads in part: "The Wolf Foundation Prize for Mathematics in 2018 will be awarded to Professors Alexander Beilinson and Vladimir Drinfeld, both of the University of Chicago, for their groundbreaking work in algebraic geometry (a field that integrates abstract algebra with geometry), in mathematical physics and in representation theory, a field which helps to understand complex algebraic structures. An 'algebraic structure' is a set of objects, including the actions that can be performed on those objects, that obey certain axioms. One of the roles of modern algebra is to research, in the most general and abstract way possible, the properties of various algebraic structures (including their objects), many of which are amazingly complicated."

Beilinson's "outstanding achievements include proofs of the Kashdan-Lustig and Jantzen conjectures, which play a key role in the representation theory, the development of important conjectures ('Beilinson's conjectures') in algebraic geometry, and a significant contribution to the interface between geometry and mathematical physics. The joint work of Beilinson and Vladimir Drinfeld on the Langlands Program-a woven fabric of theorems and conjectures designed to link key areas of mathematics-has led to impressive progress in

For permission to reprint this article, please contact: reprint-permission@ams.org.

DOI: http://dx.doi.org/10.1090/noti1693 implementing the program in important areas of physics, such as quantum field theory and string theory.

"Drinfeld has contributed greatly to various branches of pure mathematics, mainly algebraic geometry, arithmetic geometry, and the theory of representation-as well as mathematical physics. The mathematical objects named after him include the 'Drinfeld modules,' the 'Drinfeld chtoucas,' the 'Drinfeld upper half plane,' the 'Drinfeld associator'-and so many others that one of his endorsers jokingly said, 'one could think that "Drinfeld" was an adjective, not the name of a person.'

"In the seventies, Drinfeld began his work on the aforementioned 'Langlands Program,' the ambitious program that aimed at unifying the fields of mathematics. By means of a new geometrical object he developed, which is now called 'Drinfeld chtoucas,' Drinfeld succeeded in proving some of the connections that had been indicated by the Langlands Program. In the 1980s he invented the concept of algebraic 'quantum group,' which led to a profusion of developments and innovations not only in pure mathematics but also in mathematical physics (for example, in statistical mechanics).

"Drinfeld and Beilinson together created a geometric model of algebraic theory that plays a key role in both field theory and physical string theory, thereby further strengthening the connections between abstract modern mathematics and physics. In 2004 they jointly published their work in a book [Chiral Algebras, AMS], that describes important algebraic structures used in quantum field theory, which is the theoretical basis for the particle physics of today. This publication has since become the basic reference book on this complex subject."

\section{Biographical Sketches}

Alexander Beilinson was born in 1957 in Moscow and received his $\mathrm{PhD}$ in 1988 from the Landau Institute of Theoretical Physics. He was a researcher at the Landau Institute from 1987 to 1993 and professor of mathematics at the Massachusetts Institute of Technology from 1988 to 1998 , when he moved to his present position at the 


\section{AMS COMMUNICATION}

University of Chicago. He was awarded the Moscow Mathematical Society Prize in 1985 and the Ostrowski Prize in 1999. He was elected to the National Academy of Sciences of the USA in 2017.

Vladimir Drinfeld, born in 1954 in Kharkiv, Ukraine, received his Candidate Sci. (Soviet equivalent of $\mathrm{PhD}$ ) from Moscow University in 1978. From 1978 through 1980 he was assistant professor at Bashkir University, then held a number of research positions at the Institute for Low Temperature Physics in Kharkiv until accepting a professorship at the University of Chicago in 1999. He was awarded a Fields Medal in 1990. He is a member of the Ukrainian Academy of Sciences, the National Academy of Sciences of the USA, and the American Academy of Arts and Sciences.

\section{About the Prize}

The Wolf Prize carries a cash award of US $\$ 100,000$. The science prizes are given annually in the areas of agriculture, chemistry, mathematics, medicine, and physics. Laureates receive their awards from the President of the State of Israel in a special ceremony at the Knesset Building (Israel's Parliament) in Jerusalem. The list of previous recipients of the Wolf Prize in Mathematics is available on the website of the Wolf Foundation:

www.wolffund.org.i1.

-Elaine Kehoe

\section{Photo Credits}

Photo of Alexander Beilinson by Irene Ogievetskaya.

Photo of Vladimir Drinfeld courtesy of Vladimir Drinfeld.

Computer Vision

\section{February 4 - May 10, 2019}

\section{ORGANIZING COMMITTEE}

Yali Amit, University of Chicago

Ronen Basri, Weizmann Institute of Science

Alex Berg, University of North Carolina

Tamara Berg, University of North Carolina

Pedro Felzenszwalb, Brown University

Stuart Geman, Brown University

Basilis Gidas, Brown University

David Jacobs, University of Marlyland

Benar Svaiter, IMPA

Olga Veksler, University of Western Ontario

\section{PROGRAM DESCRIPTION}

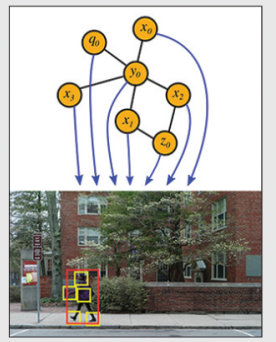

Computer vision is an inter-disciplinary topic crossing boundaries between computer science, statistics, mathematics, engineering and cognitive science. Its research involves the development and evaluation of computational methods for image analysis. This includes the design of new theoretical models and algorithms, and practical implementation of these algorithms using a variety of computer architectures and programming languages. The methods under consideration are often motivated by generative mathematical models of the world and the imaging process. Recent approaches also rely heavily on machine learning techniques and discriminative models such as deep neural networks.

The focus of the program will be on problems that involve modeling, machine learning and optimization. The program will also bridge a gap between theoretical approaches and practical algorithms, involving researchers with a variety of backgrounds.

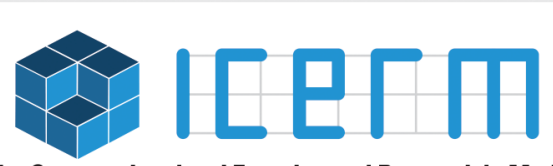

Institute for Computational and Experimental Research in Mathematics

Proposals being accepted: Semester Program

Topical/Hot Topics Workshops

Small Group Research Program

Summer Undergrad Program

ICERM is a National Science Foundation Mathematics Institute at Brown University in Providence, RI.
Appications being accepted: Semester Program or Workshop Postdoctoral Fellowship Sponsorships being accepted: Academic or Corporate

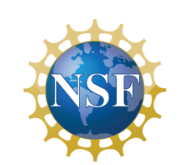

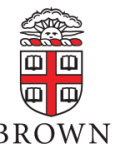

BROWN

\section{icerm.brown.edu}

